\title{
Phase Field Crystals as a Coarse-Graining in Time of Molecular Dynamics
}

\author{
P. F. Tupper ${ }^{1}$ and Martin Grant ${ }^{2}$ \\ Department of Mathematics and Statistics, McGill University, Canada. \\ Department of Physics, McGill University, Canada.
}

PACS 05.70.Ln - Nonequilibrium and irreversible thermodynamics

PACS 64.70.D- - Solid-liquid transitions

PACS 61.50.Ah - Theory of crystal structure, crystal symmetry; calculations and modeling

\begin{abstract}
Phase field crystals (PFC) are a tool for simulating materials at the atomic level. They combine the small length-scale resolution of molecular dynamics (MD) with the ability to simulate dynamics on mesoscopic time scales. We show how PFC can be interpreted as the result of applying coarse-graining in time to the microscopic density field of molecular dynamics simulations. We take the form of the free energy for the phase field from the classical density functional theory of inhomogeneous liquids and then choose coefficients to match the structure factor of the time coarse-grained microscopic density field. As an example, we show how to construct a PFC free energy for Weber and Stillinger's two-dimensional square crystal potential which models a system of proteins suspended in a membrane.
\end{abstract}

Introduction. - Phase field crystals (PFC) were introduced in [1] as a tool for performing long-time simulation of materials at the microscopic scale. The approach is -ạn example of the phase field approach to non-equilibrium modeling and simulation. The system of interest is represented by a field corresponding to the atomic density of the material. Its dynamics are simulated dissipatively ac- cording to free energy minimization with additional noise. The advantage of PFC over other phase field models is its ability to naturally resolve microscopic structure: the PFC field corresponding to a solid in equilibrium has a periodic structure matching that of the crystal stucture of the material. The advantage of PFC over molecular dynamics (MD) is that fast degrees of freedom are not present in the model. This feature allows efficient simulation over mesocopic time scales. The PFC approach can model elastic and plastic deformation of crystals as well as the liquid-solid transition [2] and diffusion of defects [3].

Previous work [2] demonstrated how phase field crystals could be constructed that matched the first peak in the structure factor of a two-dimensional hexagonal crystal material and demonstrated their use for the simulation of a variety of non-equilibrium phenomenon. Here we will accomplish two things: (i) show that phase-field crystals can be interpreted as the result of applying coarse-graining in time to microscopic molecular dynamics (ii) show how to generalize phase-field crystals in order to model different materials and match more than the first peak in the structure factor. The system we will use as an example for this is Weber and Stillinger's [4] two-dimensional square-crystal forming system. This system was originally proposed as a model of various systems where square twodimensional crystals occur, including proteins suspended in a membrane and planar levels in colloidal suspensions. We will show that we can obtain a PFC free energy that matches arbitrarily many peaks of the stucture factor of the time-coarsened microscopic density for this potential.

Coarse-Graining Molecular Dynamics. - We consider a system of $N$ particles in the plane each with mass $m$ and with positions $r_{i}$ and momenta $p_{i}, i=$ $1, \ldots, N$. The energy of the system is given by

$$
H_{N}=\sum_{i=1}^{N} \frac{p_{i}^{2}}{2 m}+V\left(r_{1}, \ldots, r_{N}\right) .
$$

The $V$ used by Weber and Stillinger [4] contains both twobody and three-body terms:

$V\left(r_{1}, \ldots, r_{N}\right)=\sum_{i \neq j} v^{(2)}\left(\left|r_{i}-r_{j}\right|\right)+\lambda \sum_{i, j, k} v^{(3)}\left(r_{i}, r_{j}, r_{k}\right)$. 
The pair potential is given by

$v^{(2)}(r)= \begin{cases}A\left(r^{-12}-r^{-5}\right) \exp \left[(r-a)^{-1}\right], & 0<r<a \\ 0, & r \geq a,\end{cases}$

where $A=6.767441, a=2.46491832$. The three-body term is given by

$$
\begin{aligned}
v^{(3)}\left(r_{i}, r_{j}, r_{k}\right) & =h\left(r_{i j}, r_{j k}, \theta_{i}\right)+h\left(r_{j i}, r_{j k}, \theta_{j}\right) \\
& +h\left(r_{k i}, r_{k j}, \theta_{k}\right),
\end{aligned}
$$

where $r_{i j}=\left|r_{i}-r_{j}\right|$ and $\theta_{i}$ is the angle between $r_{j}-r_{i}$ and $r_{k}-r_{i}$. The function $h$ is

$$
h(r, s, \theta)=\exp \left[\left(r-a_{3}\right)^{-1}+\left(s-a_{3}\right)^{-1}\right] \sin ^{2}(2 \theta),
$$

when $r, s<a_{3}$, and is zero otherwise. Weber and Stillinger choose $a_{3}=1.8$ and $\lambda=5$. Without the threebody terms the potential is qualitatively the same as the Lennard-Jones potential, and the system has a hexagonal crystal phase like that modeled by the PFC in $[2,3]$. The addition of the three-body term makes hexagonal structure unfavourable and the crystal phase has square symmetry [4]. The dynamics of the system were simulated using molecular dynamics.

The microscopic density field is given by $\rho_{m}(r, t)=$ $\sum_{i=1}^{N} \delta\left(r-r_{i}(t)\right)$, where $r_{i}(t)$ is the position of the $i$ th particle at time $t$. We consider the density coarse-grained in time:

$$
\rho(r)=\frac{1}{\tau} \int_{0}^{\tau} d t \rho_{m}(r)
$$

If the original system is in the liquid phase, for large $\tau$ the field $\rho$ will be equal to a constant plus small, spatially homogenous fluctuations. If the original system is in the solid phase, $\rho$ will have a steady periodic form for large $\tau$ corresponding to the structure of the crystal. Fig. 1 shows density plots of $\rho$ of a system in solid phase for four different coarse-graining times $\tau$. As $\tau$ is increased fluctuations about equilibrium become smaller and occur over a smaller time scale.

The Phase Field Crystal. - Our goal is to obtain a phase field model for the dynamics of the coarse-grained density field $\rho$ given by eq. (2) for large but finite $\tau$. This goal can be broken into two distinct parts. Firstly, we need to determine a free energy $\mathcal{F}$ functional whose minimum is a good approximation to the equilibrium configuration of $\rho$. Secondly, we need to specify evolution equations that describe how the system approaches equilibrium.

Free Energy. The motivation for the particular free energy functional $\mathcal{F}$ we use comes from classical density functional theory $[5,6]$. The goal of Ramakrishnan and Yussouff's density functional theory is to estimate a free energy $\mathcal{F}$ for which the infinite-time averaged density $\rho=\rho_{\infty}=\left\langle\rho_{m}\right\rangle$ is a minimum. They derive this free energy formally by integrating the partition function; the calculation can only be completed in the case of the ideal gas. To obtain usable approximations they postulate that

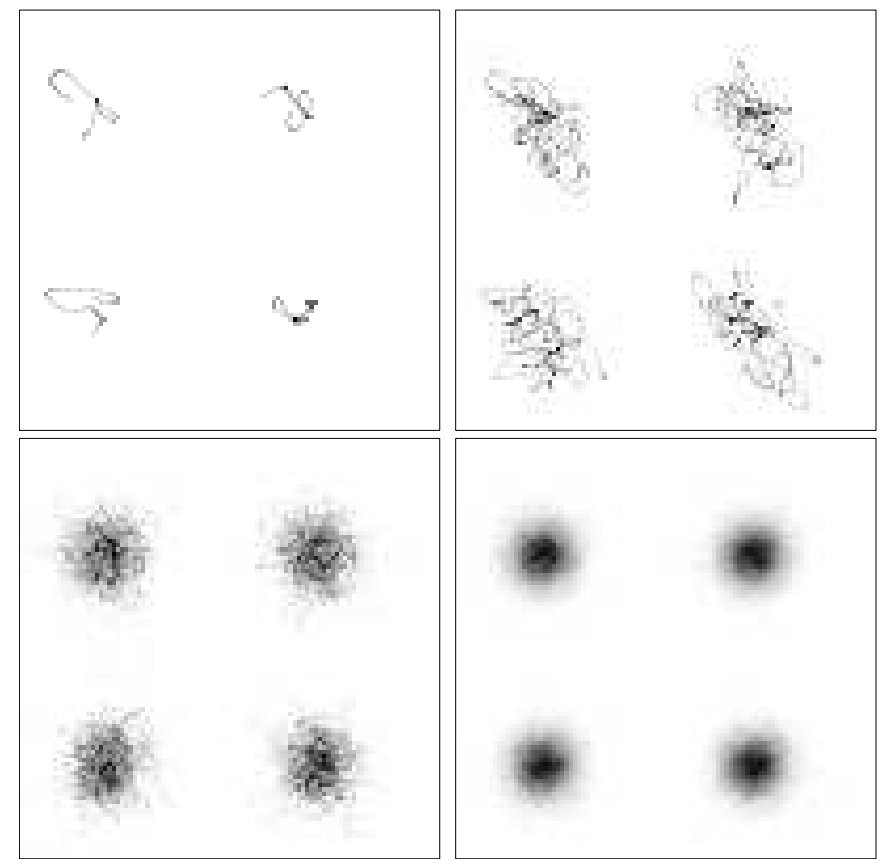

Fig. 1: Grey-scale plot of time-averaged density of portion of Weber-Stillinger MD system for reduced temperature 0.6, reduced density 0.77277 . The time intervals for averaging are 1 , 10, 100, 1000 time units.

$\rho$ has a free energy $\mathcal{F}(\rho)=\mathcal{F}_{i d}(\rho)+\mathcal{F}_{\text {ex }}(\rho)$ where $\mathcal{F}_{i d}$ is the ideal gas free energy and $\mathcal{F}_{e x}$ is a functional Taylor series expansion in $\rho$ about the uniform liquid state density [5]. Typically, only two terms in the expansion are taken for most applications [7].

Our goal is somewhat different than that of density functional theory; we are interested in densities averaged over finite times $\tau$, rather than infinite times. This allows us to model non-equilibrium phenomena. We start with free energies of density functional theory form and then add noise to account for our merely finite length of time-coarsening interval $\tau$. Moreover, rather than deriving coefficients in the free energy from liquid state equilibrium correlation functions, we choose coefficients that will be easy to compute, give the correct qualitative behaviour and have parameters that can then be tuned to match MD.

Our free energy is of the form

$$
\begin{aligned}
\mathcal{F}(\rho) & =\int d r T \rho(r)(\log (\rho)-1)-\int d r \rho(r) \mu \\
& +\frac{k_{A}}{2} \iint d r_{1} d r_{2} C_{2}\left(r_{1}, r_{2}\right) \rho\left(r_{1}\right) \rho\left(r_{2}\right) \\
& +\frac{k_{B}}{3} \iiint d r_{1} d r_{2} d r_{3} C_{3}\left(r_{1}, r_{2}, r_{3}\right) \rho\left(r_{1}\right) \rho\left(r_{2}\right) \rho\left(r_{3}\right)
\end{aligned}
$$

The last cubic term is necessary to obtain a square crystal structure and is analogous to the three-body potential term in [4]. The variable $T$ corresponds to temperature.

According to density functional theory, the kernal $C_{2}$ should be a multiple of the direct pair correlation function of the system in the liquid state [8]. Following this we 
choose our $C_{2}$ to be radially symmetic and translationally invariant, giving $C_{2}\left(r_{1}, r_{2}\right)=c\left(\left|r_{1}-r_{2}\right|\right)$ for some function $c$ that decays to zero at infinity. However, instead of matching $c$ to the correlation function exactly we instead choose a simple, compact bump function. Our results are quite insensitive to the exact details of the choice of $c$ we use, the most important quantities being the height and the location of the peak of the Fourier transform of $c$, both of which can be controlled by scaling. This is predicted by the early papers on density functional theory [5] and in later analyses tying density function theory to phase field crystals [9]. The function $c(r)=1$ for $|r|<r_{0}, c(r)=0$ otherwise works for our purposes, but in order to minimize grid artefacts we use the following smoother choice:

$$
c(r)=\frac{1}{2}\left(\frac{\arctan 20\left(r / r_{0}-1\right)}{2 \pi}-1\right) .
$$

For ease of computation, this function is then truncated at radius $2 r_{0}$ and shifted down to be continuous. We fix $r_{0}=6$ and $k_{A}=1$. Again, the $C_{3}$ from density functional theory is a three-point correlation function that could in principle be computed from molecular dynamics. Instead we choose a simple, smooth, compactly supported $C_{3}$ that gives the qualitatively correct behaviour:

$$
\begin{aligned}
C_{3}\left(r_{1}, r_{2}, r_{3}\right)= & U\left(r_{12}, r_{13}, \theta_{123}\right)+U\left(r_{21}, r_{23}, \theta_{213}\right) \\
+ & U\left(r_{31}, r_{32}, \theta_{312}\right)
\end{aligned}
$$

where $r_{i j}=\left|r_{j}-r_{i}\right|$ and $\theta_{i j k}$ is the angle between $r_{j}-r_{i}$ and $r_{k}-r_{i}$. We let

$$
U\left(r_{12}, r_{23}, \theta\right)=H\left(r_{12}\right) H\left(r_{23}\right) \sin ^{2}(2 \theta)
$$

where

$$
H(r)=\exp \left[\left(r-r_{\max }\right)^{-1}+\left(r_{\min }-r\right)^{-1}\right],
$$

for $r_{\min }<r<r_{\max }$ and $H(r)=0$ otherwise. We fix $r_{\min }=6, r_{\max }=10$ and $k_{B}=0.05$. We did not find that the resulting equilibrium configuration was sensitive to the details of $C_{3}$. The purpose of the third-order term is only to make a hexagonal pattern unstable and the square pattern more energentically favourable.

Equations for time evolution. Now that we have a form for the free energy $\mathcal{F}$, we need to state equations for the time evolution of the phase field. In the present work we do not match non-equilibrium properties of the PFC to those of MD. Instead we only want plausible dynamics with the correct equilibrium properties. In this way we can use the dynamics to sample efficiently from the equiblibrium states. Accordingly, we use a form of equation from the dynamic density functional theory of interacting Brownian particles [10]:

$$
\frac{\partial \rho(r)}{\partial t}=\Gamma \vec{\nabla} \cdot\left(\rho \vec{\nabla} \frac{\delta \mathcal{F}}{\delta \rho(r)}\right)+\vec{\nabla} \cdot\left(\sqrt{2 \sigma^{2} \Gamma} \sqrt{\rho} \vec{\eta}(r, t)\right),
$$

where $\eta(r, t)$ is a mean zero noise field with

$$
\left\langle\eta_{i}(r, t) \eta_{j}\left(r^{\prime}, t^{\prime}\right)\right\rangle=\sigma^{2} \delta_{i j} \delta\left(r-r^{\prime}\right) \delta\left(t-t^{\prime}\right) .
$$

This yields dynamics in which mass is conserved. We will also consider

$$
\frac{\partial \rho(r)}{\partial t}=-\Gamma \rho \frac{\delta \mathcal{F}}{\delta \rho(r)}+\Gamma \sigma^{2}+\sqrt{2 \sigma^{2} \Gamma} \sqrt{\rho} \xi(r, t)
$$

which corresponds to constant chemical potential. Here

$$
\left\langle\xi(r, t) \xi\left(r^{\prime}, t^{\prime}\right)\right\rangle=\delta\left(r-r^{\prime}\right) \delta\left(t-t^{\prime}\right) .
$$

In both cases the noise should be interpreted in the Ito rather than the Stratonovich sense. The $\Gamma \sigma^{2}$ term in eq. (4) does not represent an external force but is a consequence of using multiplicative noise. It is necessary for the dynamics to satisfy detailed balance, as can be checked with the Fokker-Planck equation. Both of these dynamics yield an equilibrium distribution for $\rho$ proportional to $\exp \left(-\mathcal{F}(\rho) / \sigma^{2}\right)$.

Naturally, neither of these equations are expected to correctly capture dynamics far from equilibrium and close to equilibrium they will only be accurate in situations where molecular dynamics is well matched by Brownian dynamics. The non-conserved dynamics has the same equilibrium properites as the conserved dynamics and can be simulated numerically more efficiently, so we shall use them for our studies here. We will show that for suitably chosen $\sigma$ and parameters in $\mathcal{F}$ the dynamics of eq. (40) has an equilibrium distribution that matches that of the timeaveraged microscopic density $\rho$, at a given temperature, density, and coarse-graining time $\tau$.

The resulting evolution equations when we use nonconserved dynamics eq. (4) with $\mathcal{F}$ given by (3) are

$$
\begin{aligned}
\frac{\partial \rho}{\partial t}= & -\Gamma \rho\left[T \log \rho-\mu+k_{A} \mathcal{C} \rho+k_{B} \mathcal{D}(\rho, \rho)\right] \\
& +\Gamma \sigma^{2}+\sigma \sqrt{2 \Gamma \rho} \xi,
\end{aligned}
$$

where $\mathcal{C} \rho\left(r_{1}\right)=\int d r_{2} c\left(r_{1}-r_{2}\right) \rho\left(r_{2}\right)$ and

$$
\mathcal{D}(\rho, \rho)\left(r_{1}\right)=\iint d r_{2} d r_{3} C_{3}\left(r_{1}, r_{2}, r_{3}\right) \rho\left(r_{2}\right) \rho\left(r_{3}\right) .
$$

Here $\xi$ is noise white in space and time.

Numerical Results. - To numerically simulate the dynamics $\rho$ is discretized on a rectangular grid. In the units we use, the distance between two adjacent points on the grid is 1 unit. All integrals are evaluated by straightforward summation on the grid. The equation are integrated in time using the explicit Euler method, typically with a time step of length $\Delta t=0.01$.

For particular values of the parameter $\mu$ and without noise $(\sigma=0)$ the field $\rho$ has two possible equilibria selected depending on $T$. For large $T$ the log term dominates and the stable equilibrium is a constant $\rho$ corresponding to a liquid state. As $T$ is decreased, this state becomes unstable and the system has a periodic free energy minimum resembling the fourth plot in fig. 1 With the parameters described here the distance between two adjacent bumps in the periodic phase is approximately 8.5 units. 


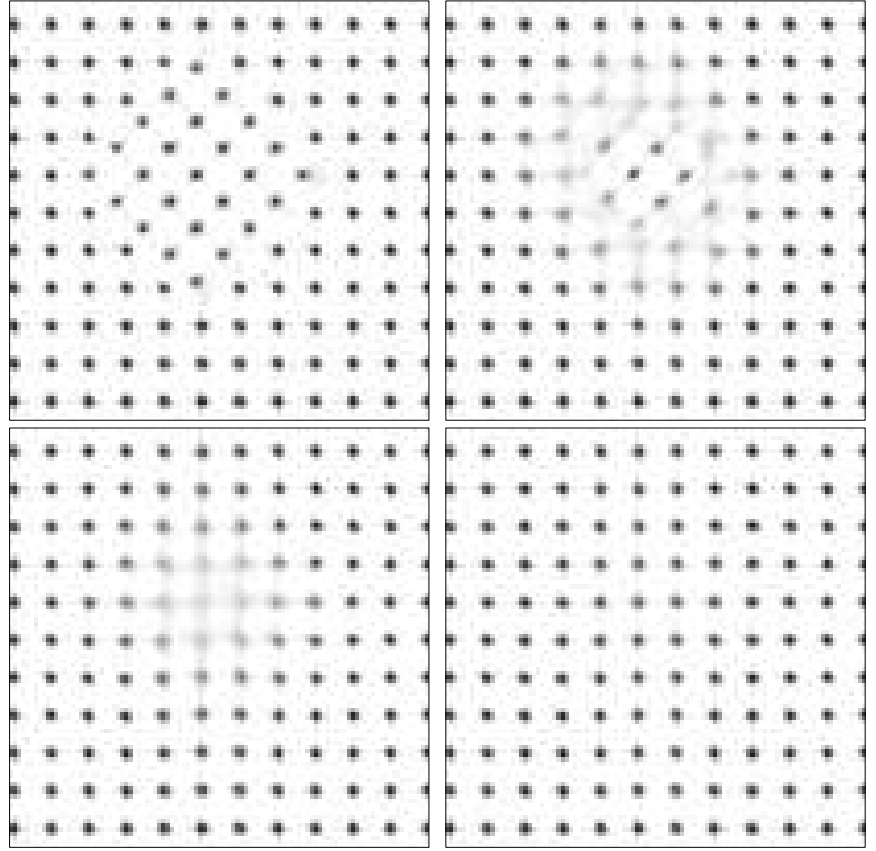

Fig. 2: Grey-scale plot of $\rho$ on a portion of the computational domain during simulation with non-conserved dynamics. Plots shown at $0,33,66,99$ time units.

Nonequilibrium simulation. Fig. 2 shows a nonequilibrium simulation of the system with non-conserved dynamics. With $\mu=10, T=2.2, \sigma=0$ the system was started in the equilibrium crystal state except for one one patch consisting of square of 25 particle bumps which is rotated $45^{\circ}$. The system was integrated in time until close to the uniform solid configuration equilibrium. In grey-scale we show density plots of the system for four representative times.

Structure Factor. We now compare results numerically obtained using this free energy with those of timeaveraged molecular dynamics. For a wide range of temperatures, densities, and time-coarsening times of the MD system, we can choose $T, \mu$ and $\sigma$ for the PFC to obtain good agreement, as we discuss further below.

Fig. 3] shows the structure factor for a time-averaged MD simulation together with the structure factor for an equilibrium PFC configuration where parameters have been chosen to obtain the best fit. The MD structure factor was taken from a 400 particle system in a perfect square crystal of density 0.77277 (leading to the minimum energy for the ground state) and reduced temperature 0.55 . The PFC was obtained from a non-conserved dynamics run with $\mu=10, T=0.8, \sigma=0.02$. The values for these parameters were chosen as follows. Since vertical and horizontal scales on the structure factor could be matched trivially be rescaling the dimensions of density and distance, we only concerned ourselves with matching the relative heights of the peaks and their relative spacing. With $\sigma=0$ (no noise) $\mu$ and $T$ were first chosen in order to ob-

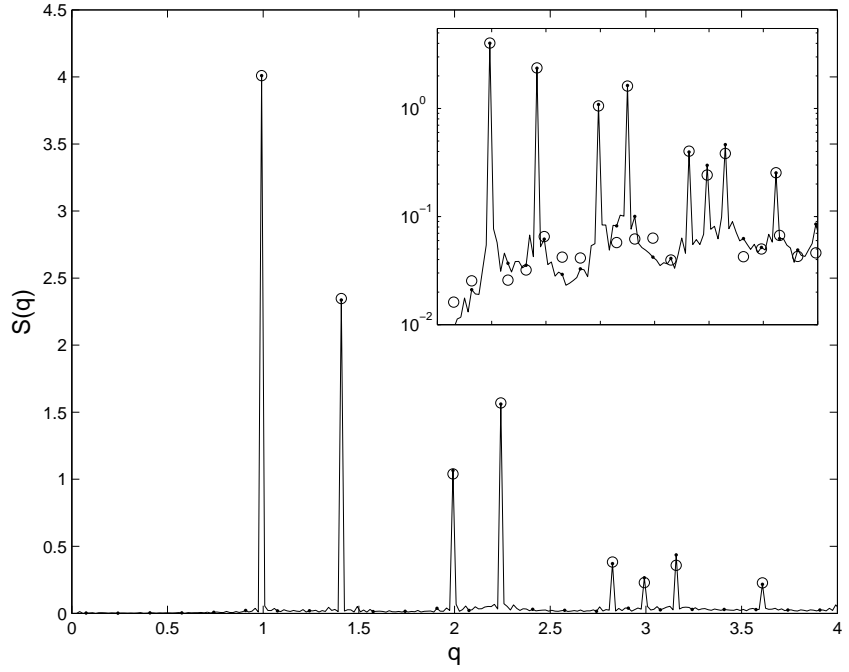

Fig. 3: Comparison of structure factor $S(q)$ versus wavenumber $q$ for MD (line and dots) and PFC (circles). For clarity only some of the data points are shown for PFC. The inset shows the same data on a logarithmic scale.

tain a square crystal pattern. This occurs for a broad range of these parameters and immediately gives the correct spacing of peaks in the structure factor. No further adjustments of $\mu$ were necessary. The value of $T$ was then adjusted to match the ratio of the first two peaks of the structure factor to that of the MD results. As $T$ goes to zero (a perfect crystal) the relative height of the second peak grows, and as $T$ goes to the melting point the second peak shrinks. As soon as the second peak was mathced all the remaining peaks agreed to the degree shown in Fig. 3 , At this point the structure factor of PFC consists purely of peaks and it was necessary to increase $\sigma$ until the background rose to match that of the MD structure factor's background.

Melting Transition. An open question in the theory of phase transitions is whether the melting transition form solid to liquid in two-dimensional systems occurs via a single phase transition or if there are two phase transitions with an intermediate hexatic phase [11]. Weber and Stillinger studied this question for their system using MD, and found no evidence for a two-stage melting process [4]. We perform an analogous experiment using our PFC model of the system. As in [4], we examine the liquid-solid transition by starting the system in a perfect square crystal and increasing the temperature (in our case, the parameter $T$ ) slowly. We used the non-conserved dynamics with $\mu=30$, and $\sigma=0,0.01,0.02,0.04$. A phase transiton should appear in a plot of mean density versus temperature as a discontinuity in the solution or its derivative. In order to highlight any such points of non-smoothness, we first smooth the mean density as a function of $T$ and then consider its derivative with respect to $T$. In fig. 4 we show the negative derivative of mean density $\bar{\rho}$ with respect to $T$ as a function of $T$. We observe only one peak in the deriva- 


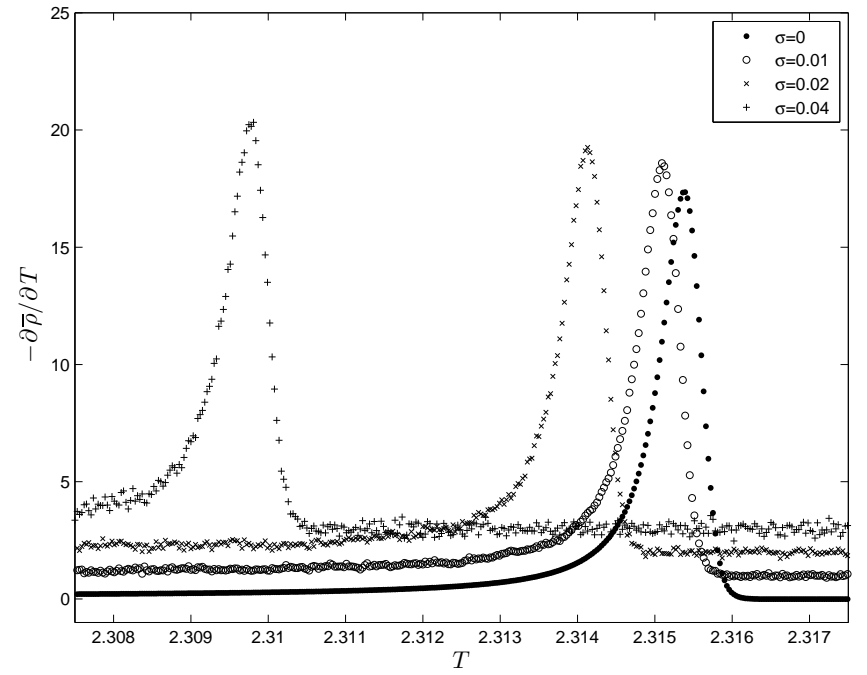

Fig. 4: Plot of $-\partial \bar{\rho} / \partial T$ as a function of $T$ for the PFC system, where $\bar{\rho}$ is mean density and $T$ is temperature. Results are shown for four different values of the noise strength $\sigma$. Plots are offset vertically from zero for clarity.

tive for each run of the simulation, suggesting a one-stage melting process. Our results are consistent with those of Weber and Stillinger.

This work was supported by the National Science and Engineering Research Council and le Fonds québécois de la recherche sur la nature et les technologies. The authors thank Ken Elder, Sami Majaniemi, and Dan Vernon for helpful discussions.

\section{REFERENCES}

[1] K. R. Elder, M. Katakowski, M. HaAtaja and M. Grant, Phys. Rev. Lett., 88 (2002) 245701.

[2] K. R. Elder and M. Grant, Phys. Rev. E, 70 (2004) 051605.

[3] J. Berry, M. Grant and K. R. Elder, Phys. Rev. E, 73 (2006) 031609.

[4] T. A. Weber and F. H. Stillinger, Phys. Rev. E, 48 (1993) 4351.

[5] T. V. Ramakrishnan and M. Yussouff, Phys. Rev. B, 19 (1979) 2775.

[6] A. D. J. Haymet and D. W. Oxtoby, J. Chem. Phys., 74 (1981) 2559.

[7] Y. Singh, Phys. Rep., 6 (1991) 351.

[8] P. M. Chaikin and T. C. Lubensky, Principles of condensed matter physics (Cambridge University Press) 1995, pp. $195-198$

[9] K. R. Elder, N. Provatas, J. Berry, P. Stefanovic and M. Grant, Phys. Rev. B, 75 (2007) 064107

[10] A. J. Archer and M. Rauscher, J. Phys. A: Math. Gen., 37 (2004) 9325.
[11] David R. Nelson, Defects and Geometry in Condensed Matter Physics (Cambridge University Press) 2002, sect. 2.4 